\title{
Rent A Trend
}

\author{
Atik Zakirhusen Mujawar, Akash Lalitkumar Makwana, Lalit Shailesh Jain, Dev Vikesh Doshi, Smita Bansod \\ IT Department, Shah and Anchor Kutchhi, Engineering College Mumbai, Maharashtra, India
}

\begin{abstract}
Article Info

Volume 7, Issue 6

Page Number: $44-48$

\section{Publication Issue :}

November-December-2021

\section{Article History}

Accepted : 10 Nov 2021

Published : 21 Nov 2021

This paper presents the execution of an on the web design rental help. It very well may be arranged as an online stage where individuals can lease things with practically no issue. It employments an extremely proficient way of advancing employing selective dresses and assistants to individuals who doesn't possess the ability to claim costly items or are less keen on claiming for different reasons.

People are more or less familiar with buying and selling clothes, but have not really experienced clothes renting or reselling online. A web-based platform where people can easily rent fashion things will be a great exposure. Useful for people who are not able to own expensive products for various reasons. The system is designed in a way that takes care of the user by providing accurate information about the product. We aim to create an application that will provide a one stop solution for all fashion related problems.
\end{abstract}

Keyword - HTML, CSS, JAVASCRIPT, DJANGO, ECOMMERCE WEBSITE, RENT, BUY, FASHION

\section{INTRODUCTION}

This website is a reliable stage that can advance style rental and make the current framework more advantageous and easier to understand. We intend to refresh the current framework by making it electronic, giving a straightforward UI and making it financially savvy. Individuals who need to lease the garments wouldn't have to go to rental shops to lease garments. They just need to peruse the framework and give some data. Framework would assist the clients with understanding which item would address their issue best. Application will add a better approach for life by giving every one of the offices that anybody can demand in the style exchanging and rental framework. Our website follows the following procedure, firstly the user has to login/register, then he/she will be will directed to the landing page where he can see the men and women section and the various categories mentioned. $\mathrm{He} / \mathrm{she}$ then after selecting that category will be directed to that page where all the products related to that category will be displayed to him, he/she can then select the product they like and want to purchase, after selecting they can put that product in the cart. After finishing the shopping and putting all the required products in the cart they can check out by making the payment which is a reliable. 


\section{LITERATURE REVIEW}

In the paper [1] technology used was Data Mining, there were different techniques mentioned such as Affinity analysis, logistic regression, Linear regression, sales strategy. The database containing all the data of the sold products, product selling patterns, stocks of the products price, were its prime features. Its drawback is the data breach can happen in the system.

This paper [2] is implemented using Deep Learning and includes technologies like disjoint set sampling and data cleansing. The main goal is to build a machine learning system that accepts a variable number of items yet produce a consistent score for any size of combination.

The basic features of the paper [3] was to build an application where user can buy, rent, repair and rent cars. Technologies used were HTML, CSS in Frontend and Django in Backend. Authentication was the main problem in this system.

The paper[6] had used technologies like OpenCart and PhpStudy, MYSQL. Different techniques were MVC architecture, Apriori algorithm. Some of the features were specialties of Fujian, Analysis, Apriori algorithm.

In the paper [8] technology used HTML, CSS, JAVASCRIPT. Interactive virtual try-on can also be an interesting feature of digital signage for advertisement and/or attracting crowds, Consumer Acceptance of Electronic Commerce questionnaire, Consumer Acceptance of Electronic Technology Acceptance Model (TAM). It has a disadvantage as Usage of orthodox and boring method of questionnaires.

The paper[7] had used technologies like HTML, CSS, JAVASCRIPT. Different techniques were Hypertext
Transfer Protocol (HTTP), profile generator, bill maker, receipt generator. Some of the features were Users can rent products from big fashion house so they the purchase is fulfilled without even actually purchasing the products and drawback is Absence of rating system.

In the paper [4] technology used was Microsoft Kinect [14], Image processing. Its Features where It can enhance the user's shopping experience through new features, such as side-by-side comparison of various clothes and simultaneous viewing of outfits from different angles. Interactive virtual try-on can also be an interesting feature of digital signage for advertisement and/or attracting crowds.

It also some drawbacks like while using the application users find the UI quite confusing also some of the users get flustered while using it.

The paper[5] had used technologies like HTML, CSS, Javascript, jQuery, Ajax, PHP, DBMS. Different techniques were Hypertext Transfer Protocol (HTTP), A registration page, Search option, Shopping cart, Admin page, Payment page, E-billing. Some of the features were Cash on delivery, based on categories and the drawbacks where Minimum quantity is 5 or request admin or supplier.

\section{EXISTING SYSTEM}

In the existing system, the entire workflow structure is conducted on papers. Information in regard to the order is hand written. Whenever the customer comes for the second time, all the previous information is written down again instead of maintaining a record of previous purchases. The bills are generated in a manual process on a separate sheet. The information related to the order is written on a piece of paper for each customer which gets to be destroyed after a period of time to decrease the paper loads. Customers have to choose from limited collections. Customers 
have to be physically present at those stores to choose from their limited collection.

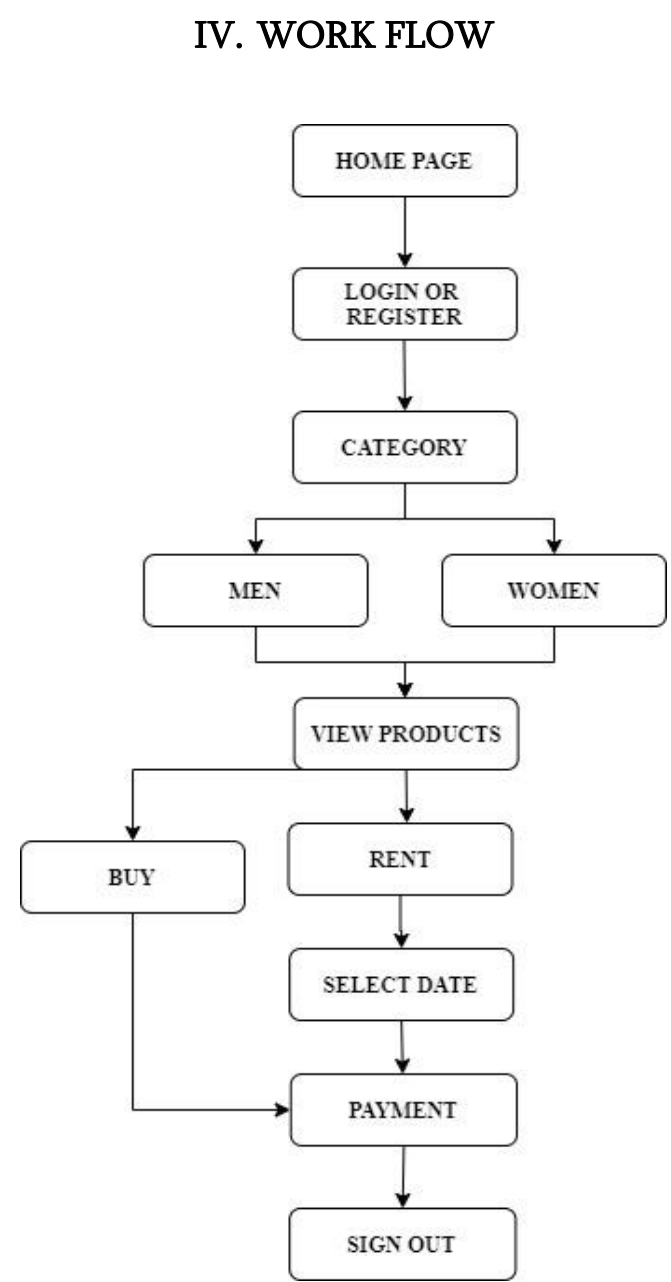

Fig. 1. System Flow

First user will see home page. User can do window shopping. If user has to buy something then he/she has to login or register then user can see the category of men and women. On clicking on any product one view its product details then rent or buy the product. If someone has to rent any product then user has to select the date on which day the user want to hire the product then the user has to make the payment after making payment the user can logout.

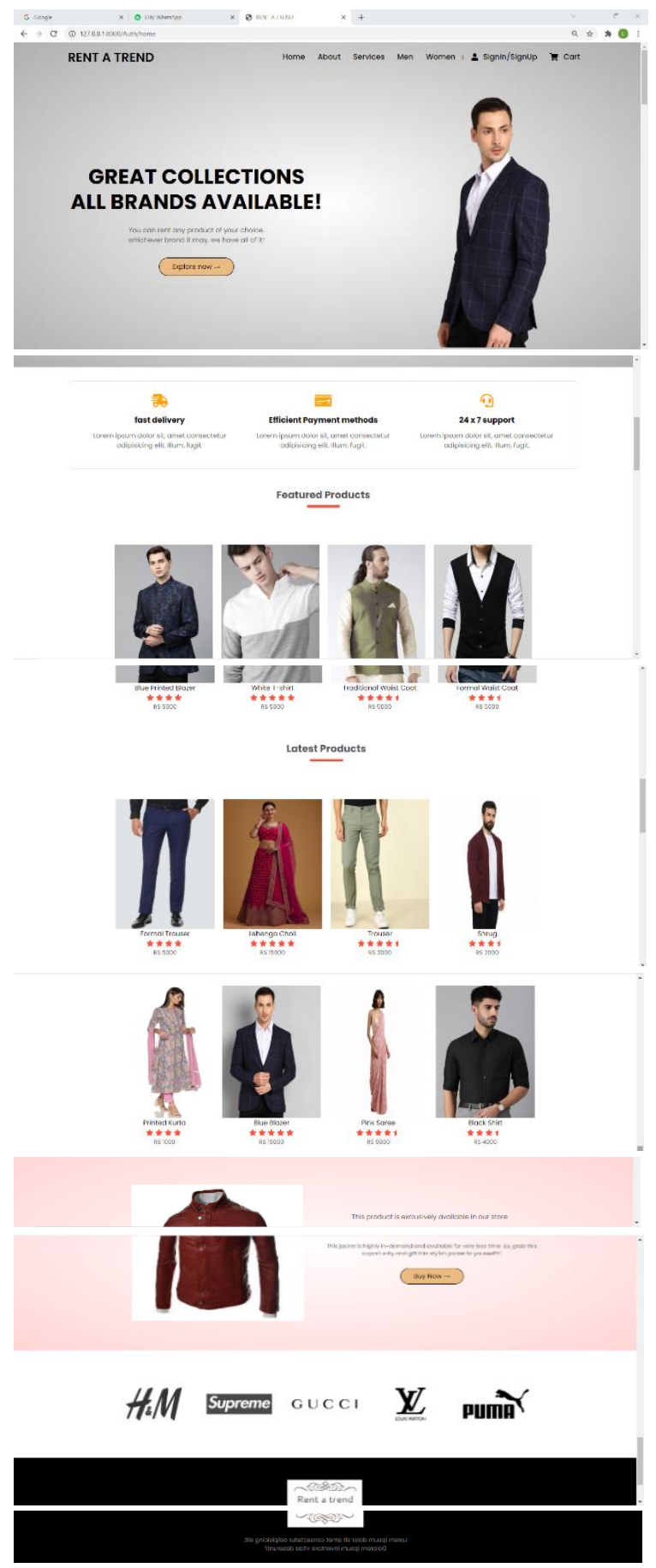

FIG 2 : HOME PAGE

This is the homepage of our website; user can see the display pictures here. And also, could get a general idea about the functioning of our website.

If user wants to rent/buy the product, so he must login through this page. If he/she is not registered he/she can sign up

\section{IMPLEMENTATION}



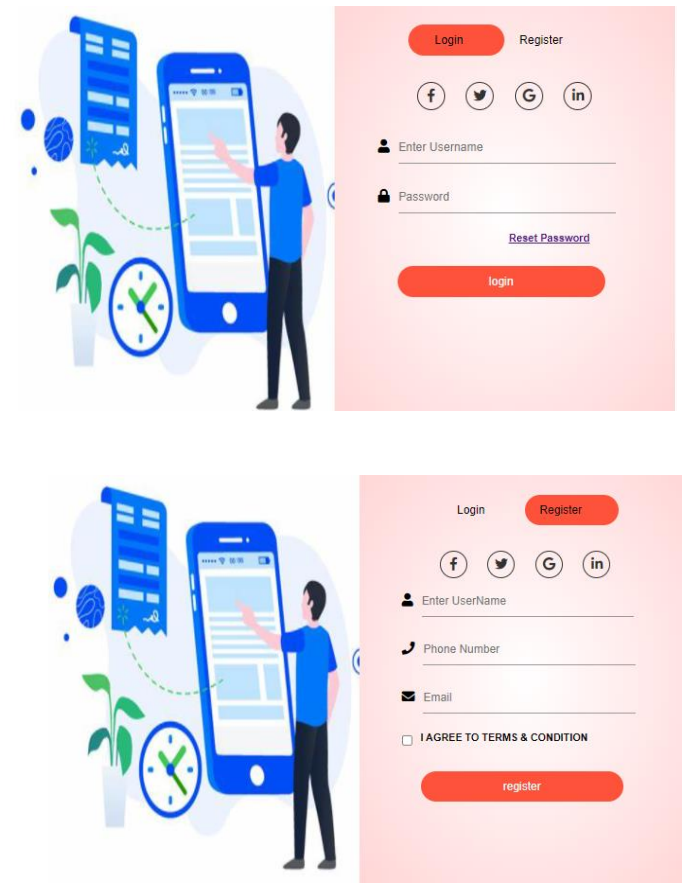

FIG3: LOGIN \& REGISTER

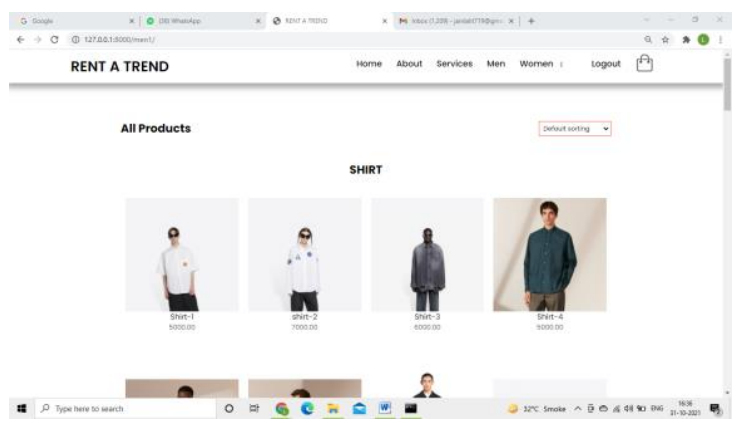

FIG4: TOP WEAR PAGE

After selecting the top-wear section, he can see the various products to buy/rent for himself

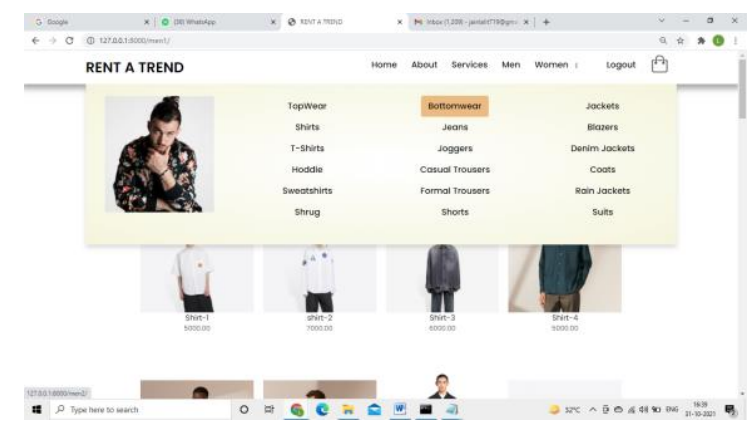

FIG5: CATEGORY

So, if the user wants to further continue on our website, he can navigate through the navbar and selects any of the option like men/women So for example If the user clicks on men the following mega-menu will appear.

\section{SUMMARY}

This paper presents the execution of an on the web design rental help. It very well may be arranged as an online stage where individuals can lease things with practically no issue. It employments an extremely proficient way of advancing employing selective dresses and assistants to individuals who doesn't possess the ability to claim costly items or are less keen on claiming for different reasons. This website is a reliable stage that can advance style rental and make the current framework more advantageous and easier to understand.

We intend to refresh the current framework by making it electronic, giving a straightforward UI and making it financially savvy. Individuals who need to lease the garments wouldn't have to go to rental shops to lease garments. They just need to peruse the framework and give some data. Framework would assist the clients with understanding which item would address their issue best. Application will add a better approach for life by giving every one of the offices that anybody can demand in the style exchanging and rental framework. After finishing the shopping and putting all the required products in the cart they can check out by making the payment which is a reliable.

\section{ACKNOWLEDGEMENT}

The satisfaction that accompanies that the successful completion of any task would be incomplete without the mention of people whose ceaseless cooperation made it possible, whose constant guidance and encouragement crown all efforts with success. We are grateful to our project guide Ms.Smita Bansod for the guidance, inspiration and constructive suggestions that helpful us in the preparation of this project. 


\section{REFERENCES}

[1]. Z. Pirani, A. Marewar, Z. Bhavnagarwala and M. Kamble, "Analysis and optimization of online sales of products," 2017 International Conference on Innovations in Information, Embedded and Communication Systems (ICIIECS), 2017, pp. 1-5, doi: 10.1109/ICIIECS.2017.8276165.

[2]. P. Tangseng, K. Yamaguchi and T. Okatani, "Recommending Outfits from Personal Closet," 2017 IEEE International Conference on Computer Vision Workshops (ICCVW), 2017, pp. 2275-2279, doi: 10.1109/ICCVW.2017.267.

[3]. S. H. Mahi, U. H. Maliha and S. Sakib, "Development of Web and Mobile Application Based Online Buy, Sell and Rent Car System," 2020 Advanced Computing and Communication Technologies for High Performance Applications (ACCTHPA), 2020, pp. 143-147, doi: 10.1109/ACCTHPA49271.2020.9213208.

[4]. M. Yuan, I. R. Khan, F. Farbiz, S. Yao, A. Niswar and M. Foo, "A Mixed Reality Virtual Clothes Try-On System," in IEEE Transactions on Multimedia, vol. 15, no. 8, pp. 1958-1968, Dec. 2013, doi: 10.1109/TMM.2013.2280560.

[5]. S. E. Ullah, T. Alauddin and H. U. Zaman, "Developing an E-commerce website," 2016 International Conference on Microelectronics, Computing and Communications (MicroCom), 2016, pp. $1-4$, doi:10.1109/MicroCom.2016.7522526.

[6]. Z. -J. Lee, S. -J. Lin, Y. -H. Guo, Y. -H. Zhang and Z. -G. Qiu, "Intelligent Online Shopping Store for Specialties of Fujian," 2020 3rd IEEE International Conference on Knowledge Innovation and Invention (ICKII), 2020, pp. 179-181, doi: 10.1109/ICKII50300.2020.9318884.

[7]. F. Soroni, M. T. Mahmud, S. Chowdhury and M. M. Khan, "RentBd-An Exclusive Fashion
Rental Service," 2021 IEEE World AI IoT Congress (AIIoT), 2021, pp. 0132-0136, doi: 10.1109/AIIoT52608.2021.9454243.

[8]. F. Y. H. Ahmed, E. B. Hazlan and M. I. Abdulla, "Enhancement of Mobile-Based Application for Vehicle Rental," 2021 IEEE 11th IEEE Symposium on Computer Applications \& Industrial Electronics (ISCAIE), 2021, pp. 163168, doi: 10.1109/ISCAIE51753.2021.9431820.

[9]. R. N. Puteri and A. Widyanti, "E-commerce of Islamic fashion product: usability and user acceptance," 2018 International Conference on Information Technology Systems and Innovation (ICITSI), 2018, pp. 143-147, doi: 10.1109/ICITSI.2018.8696004.

\section{Cite this article as :}

Atik Zakirhusen Mujawar, Akash Lalitkumar Makwana, Lalit Shailesh Jain, Dev Vikesh Doshi, Smita Bansod, "Rent A Trend", International Journal of Scientific Research in Computer Science, Engineering and Information Technology (IJSRCSEIT), ISSN : 2456-3307, Volume 7 Issue 6, pp. 44-48, November-December 2021. Available at doi $\quad$ : https://doi.org/10.32628/CSEIT21768

Journal URL : https://ijsrcseit.com/CSEIT21768 